\title{
A Novel ERCC6 Splicing Variant Associated with a Mild Cockayne Syndrome Phenotype
}

\author{
Jonathan M. Swartz ${ }^{a}$ Aysehan Akinci ${ }^{d}$ Shayne F. Andrew ${ }^{b} \quad$ Ahmet Siğirci $^{\mathrm{e}}$ \\ Joel N. Hirschhorn ${ }^{a}$ Ron G. Rosenfeld ${ }^{c}$ Andrew Dauber ${ }^{b}$ Vivian Hwa ${ }^{b}$ \\ ${ }^{a}$ Division of Endocrinology, Boston Children's Hospital, Boston, Mass., b Division of Endocrinology, Cincinnati Children's \\ Hospital Medical Center, Cincinnati, Ohio, and 'Department of Pediatrics, Oregon Health and Science University, Portland, \\ Oreg., USA; Departments of d Pediatric Endocrinology and ePediatric Radiology, Inonu University, Turgut Özal Medical \\ Center, Malatya, Turkey
}

\section{Established Facts}

- Molecular defects in 2 genes, ERCC6 and ERCC8, are associated with Cockayne syndrome, with around $80 \%$ of affected individuals having mutations in ERCC6.

- To date, at least 78 mutations have been identified in ERCC6, including 12 splicing mutations. There is significant variability in the severity of cases and ongoing debate over genotype-phenotype correlations.

- Phenotypic variability in Cockayne syndrome leads to challenges in establishing a diagnosis, particularly in nonclassical cases.

\section{Novel Insights}

- Whole-exome sequencing performed on an extended family led to the diagnosis of Cockayne syndrome, with significant intrafamily variability in phenotypic features.

- The identified novel homozygous ERCC6 c. $1992+3 A>G$ splicing variant demonstrates the significant functional and biological impact of a variant computationally predicted to cause only a minor change in the relative strength of a splice site.

\section{Key Words}

ERCC6 variant $\cdot$ Cockayne syndrome $\cdot$ Short stature

\section{Abstract \\ Background: Cockayne syndrome is an autosomal recessive, heterogeneous syndrome with classical features, including short stature, microcephaly, developmental delay, neuropa-}

thy, and photosensitivity. New genomic approaches offer improved molecular diagnostic potential. Methods: Wholeexome sequencing was employed to study a consanguineous extended family with severe short stature and variable

Jonathan M. Swartz and Aysehan Akinci contributed equally to this work.

\section{KARGER}

E-Mail karger@karger.com www.karger.com/hrp (c) 2014 S. Karger AG, Basel

$1663-2818 / 14 / 0825-0344 \$ 39.50 / 0$
Vivian Hwa

Division of Endocrinology, Cincinnati Children's Hospital

Medical Center, 3333 Burnet Ave

Cincinnati, OH 45229 (USA)

E-Mail vivian.hwa@cchmc.org 
presentations of peripheral neuropathy, lipoatrophy, photosensitivity, webbed neck, and hirsutism. Results: We identified a novel homozygous ERCC6 variant at the donor splice site of intron $9(c .1992+3 A>G)$, which was predicted to only slightly perturb splicing efficiencies. Assessment of primary fibroblast-derived mRNAs, however, revealed a dominant splicing species that utilized an unsuspected putative donor splice site within exon 9, resulting in predicted early protein termination (p.Arg637Serfs*34). Conclusions: We describe a new splicing ERCC6 defect causal of Cockayne syndrome. The application of exome sequence analysis was integral to diagnosis, given the complexity of phenotypic presentation in the affected family members. The novel splicing defect, furthermore, illustrates how a seemingly minor change in the relative strength of a splice site can have significant biological consequences.

(c) 2014 S. Karger AG, Basel

\section{Introduction}

Cockayne syndrome [1] is an autosomal recessive, progressive disease, typically characterized by short stature, microcephaly, developmental delay, neuropathy, premature aging, and photosensitivity. The clinical phenotype is highly variable, ranging from severe prenatal forms to mild cases with late presentations [2]. 'Classical' Cockayne syndrome (also known as CS I), typically includes severe growth failure and cognitive/neurodevelopmental abnormalities, along with additional features including sensorineural hearing loss, retinopathy, cataracts or other ocular involvement, poor dentition, photosensitivity, and a characteristic cachectic appearance due to lipoatrophy. The average life expectancy is 16 years, but some individuals can survive into early adulthood. Cockayne syndrome II (also called CS II) is the more severe form, often notable for low birth weight with poor subsequent growth and cognitive development. Presentations are at an earlier age, with death often occurring by the age of 5-7 years. Milder forms of Cockayne syndrome have been described, including those that do not present until adolescence and where patients live to an average age of 30 years (referred to as CS III) and those that present with adult onset disease and lack many of the typical features [3-6]. The clinical spectrum, therefore, has continued to broaden and is complicated by overlapping features with other syndromes such as cerebro-oculo-facio-skeletal syndrome and UV-sensitive syndrome [7].

Molecular defects in 2 genes, ERCC6 (CSB) and ERCC 8 (CSA), have been associated with Cockayne syndrome. It has been estimated that around $80 \%$ of Cockayne syndrome patients carry mutations in ERCC6 [3], with over 78 mutations described to date [5]. ERCC6 (chromosome 10q11.23) encodes for CSB, a protein of 1,493 amino acid residues that is a member of the SNF2/SW12 family of ATPases, a subfamily of the helicase superfamily best known for its ability to regulate chromatin structure by hydrolyzing ATP to alter DNA-protein contacts [8]. Structurally, the central ATPase domain of CSB (residues 510-960) consists of 7 conserved helicase motifs which, interestingly, do not have helicase activities. A variety of DNA substrates (including double-stranded DNA fragments), however, have been shown to stimulate ATPase activity, supporting the role of CSB in DNA repair and transcriptional regulation [7-9].

Despite the large number of ERCC6 mutations [5] already ascribed to Cockayne syndrome, genotype-phenotype correlations remain to be fully elucidated, with some studies suggesting that variants leading to an absence of protein tend to have milder phenotypes than variants resulting in abnormal protein expression/functions $[4,10]$. We describe, in this report, a family with a number of affected individuals not initially recognized as presenting with Cockayne syndrome but who share a common phenotype of severe short stature. Through whole-exome sequence analyses, we identified a novel homozygous splicing defect in ERCC6. The mild and variable phenotypic features of the subjects in this study did not fit the typical clinical criteria for Cockayne syndrome, making the diagnosis challenging without the benefit of comprehensive genetic testing. Individuals in this family provide examples of the increasingly common genetic diagnoses of nonclassical and/or mild cases of syndromes that otherwise would not necessarily be readily identified by standard evaluation.

\section{Patients and Methods}

\section{Subjects}

We studied a Turkish extended family consisting of 3 affected siblings born from consanguineous parents, as well as 3 of their first cousins with significant short stature (standard deviation score, SDS, between -2.9 and -5 ). An SDS less than or equal to -2 was used to define short stature. A fourth cousin was clinically unaffected. The parents were of normal stature. Clinical evaluation did not lead to a unifying diagnosis for this family. Severe short stature was consistent among the group, but other phenotypic features were variably present in the affected family members, including intellectual impairment, peripheral neuropathy, lipoatrophy, severe photosensitivity, calcifications of the basal ganglia, and cerebellar atrophy. Additional features not typically associated with 




Fig. 1. Pedigree of family carrying the ERCC6 variant: 3 generations are represented, with family members labeled numerically. $\mathrm{WT}=$ Wild-type allele (c.1992 + 3A). Circles indicate female family members; squares indicate male family members. Black symbols denote clinically affected family members, split black symbols

Cockayne syndrome were seen in some of the affected individuals, including webbed neck, hirsutism and low posterior hairline.

The index patient (III-9; fig. 1) was evaluated at the age of 12.75 years. At the time, she was $128 \mathrm{~cm}$ tall (SDS -3.25). Her body weight was quite low (SDS -3.5), as was her BMI (SDS -2.8). She had been small since birth at 38 weeks of gestation, when she weighed $2.2 \mathrm{~kg}$. She had been on a normal diet without any supplementation or extra nutritional support. She was found to be cognitively impaired, with an estimated IQ of 70-80. She had photosensitivity, lipoatrophy, bilateral peripheral neuropathy, and hyperactivity. Her brain MRI was notable for cerebellar and optic nerve atrophy, along with basal ganglia calcifications (online suppl. fig. 1; for all online suppl. material, see www.karger.com/ doi/10.1159/000368192). Demyelination of the periventricular white matter at the centrum semiovale and subcortical area were noted. She had menarche at age 13 . She did not have any abnormal skeletal findings on examination. During the course of her workup and evaluation, she was treated with growth hormone $(\mathrm{GH})$ and grew $4.4 \mathrm{~cm}$ during the first year on treatment. Patient III-6, the sister of III-9, had a very similar phenotype, with the most notable difference being the presence of shortened 4th metacarpal and metatarsal bones in III-6. In contrast, their brother (III-8) was found to have isolated short stature with an SDS score of -4.5 at age 20 , but without any other abnormal findings (table 1 ).

One of the cousins of the proband (III-3) was initially evaluated at age 11.5 years. At the time she was $114 \mathrm{~cm}$ tall (SDS -4.5). Her body weight was less than the 3rd percentile, and her BMI was show asymptomatic carriers and symbols with a question mark indicate family members whose genotype is unknown (though not reported to have clinical syndrome). Genotype of variant is denoted. The proband is indicated by the arrow.

15.7 (SDS -1.2). She was noted to have some physical findings similar to a Turner syndrome phenotype, including a short, webbed neck, low posterior scalp hair line, cubitus valgus, and inverted nipples. Her karyotype, however, was normal. The only skeletal finding of note was short metacarpal bones. She had photosensitivity, as well as lipoatrophy, similar to her cousins. She also had hirsutism, polycystic ovarian syndrome and mildly elevated androgen levels. She did not have any neurological deficits, and her IQ was estimated between $80-85$. Her brain MRI was notable for minimal demyelination and calcification of the basal ganglia. She was treated with $\mathrm{GH}$ for 6 months with poor response (growth of $1.5 \mathrm{~cm}$ ). One of her sisters (III-1) had very similar features, but did not have shortened metacarpals. Their sister (III-4) had short stature (table 1), a short, webbed neck and low posterior scalp hair line. She did not present with intellectual deficits, neurological findings or brain MRI changes nor did she have photosensitivity or bony abnormalities (table 1).

\section{Genetic Analysis}

Peripheral blood leukocytes were obtained from available family members, and genomic DNA was extracted for analysis. Wholeexome sequencing was completed at the Broad Institute (Cambridge, Mass., USA) on 5 individuals from this family. Agilent's SureSelect human all exon kit version 2 (Agilent Technologies, Santa Clara, Calif., USA) was used for hybrid selection. Sequencing was completed for the 5 subjects on an Illumina HiSeq platform (Illumina Inc., San Diego, Calif., USA). The sequencing reads were 
Table 1. Stature data (most recent information) and clinical descriptions

\begin{tabular}{|c|c|c|c|c|c|c|c|c|}
\hline III-3 & $\mathrm{F}$ & 11 & -4.5 & $\begin{array}{l}\text { Webbed neck, low } \\
\text { posterior hairline, } \\
\text { hirsutism, polycystic } \\
\text { ovary syndrome; } \\
46, \mathrm{XX}\end{array}$ & $\begin{array}{l}\text { No neurological } \\
\text { deficits, brain MRI } \\
\text { with basal ganglia } \\
\text { calcifications, minimal } \\
\text { white matter } \\
\text { demyelination }\end{array}$ & $80-85$ & $\begin{array}{l}\text { Photosensitivity, } \\
\text { facial freckling, } \\
\text { dry skin and } \\
\text { hair, lipoatrophy }\end{array}$ & $\begin{array}{l}\text { Short } \\
\text { metacarpals, } \\
\text { limited } \\
\text { response to } \\
\text { growth } \\
\text { hormone }\end{array}$ \\
\hline III-6 & $\mathrm{F}$ & 21 & -5 & $\begin{array}{l}\text { Significant short stature, } \\
\text { born full term, SGA }\end{array}$ & $\begin{array}{l}\text { Bilateral peripheral } \\
\text { neuropathy, brain } \\
\text { MRI with cerebral, } \\
\text { cerebellar and optic } \\
\text { nerve atrophy, as well } \\
\text { as basal ganglia } \\
\text { calcifications }\end{array}$ & $75-80$ & $\begin{array}{l}\text { Severe } \\
\text { photosensitivity, } \\
\text { facial freckling, } \\
\text { lipoatrophy }\end{array}$ & $\begin{array}{l}\text { Short } 4 \text { th } \\
\text { metacarpal } \\
\text { and } \\
\text { metatarsal } \\
\text { bones }\end{array}$ \\
\hline III-8 & M & 20 & -4.5 & $\begin{array}{l}\text { Isolated short stature, } \\
\text { normal physical } \\
\text { examination }\end{array}$ & $\begin{array}{l}\text { No neurological deficits, } \\
\text { normal brain MRI }\end{array}$ & Average & Unremarkable & $\begin{array}{l}\text { Limited } \\
\text { response } \\
\text { to growth } \\
\text { hormone }\end{array}$ \\
\hline
\end{tabular}

Patient ID: refer to figure 1. WISC-R = Wechsler Intelligence Scale for Children-Revised.

aligned to the hg19 reference genome with Burrows-Wheeler Aligner [11]. The Genome Analysis Toolkit was applied for base quality score recalibration and indel (insertion-deletion) realignment [12]. Variant quality score recalibration was simultaneously performed for SNP and indel discovery and genotyping using variant quality score recalibration [13]. SnpEff (http://snpeff.source forge.net/) was used to annotate functional effect. We filtered for variants that were novel or present in less than $1 \%$ of the reference population based on allele frequency data from the 1,000 Genomes project (2/2012 release) and the National Heart, Lung, and Blood Institute (NHLBI; Bethesda, Md., USA) exome variant server (http://evs.gs.washington.edu/EVS/) as previously described [14]. The ERCC6 variant was screened by Sanger sequencing of 11 affected and unaffected family members.

\section{Analysis of ERCC6 cDNA from Primary Dermal Fibroblast} Cultures

Primary fibroblast cultures were established from skin biopsies procured from 3 affected probands and 1 carrier with consent in compliance with the institutional review board at Oregon Health and Science University. Cultures were maintained as previously described [15]. Total mRNA was extracted from untreated (DMSO) or cycloheximide-treated fibroblasts $(10 \mathrm{pmol} / \mathrm{l}$ in DMSO) [16] and reverse transcription cDNA synthesis was performed as previously reported [15]. PCR amplification of GHR (GH receptor) cDNA followed the established protocol [15]. For analysis of the relevant segment of ERCC6 CDNA (based on ERCC6 $m R N A$ NM_000124.3), the following primers were employed: forward, 5' ACAGGTGTTAGGTGGCTGTG (exon 7) and reverse, 5' 
Table 2. GHBP, IGF-1, IGFBP-3, and ALS results for multiple affected and unaffected family members

\begin{tabular}{|c|c|c|c|c|c|c|c|c|c|c|c|}
\hline $\begin{array}{l}\text { Patient } \\
\text { ID }\end{array}$ & Sex & $\begin{array}{l}\text { Age, } \\
\text { years }\end{array}$ & $\begin{array}{l}\text { Height } \\
\text { (SDS) }\end{array}$ & $\mathrm{pmol} / \mathrm{l}$ & reference & $\mathrm{ng} / \mathrm{ml}$ & reference & $\mathrm{mg} / \mathrm{l}$ & reference & $\mathrm{mg} / \mathrm{l}$ & reference \\
\hline II-2 & M & - & -0.4 & 1,157 & $686-2,019$ & 104 & $132-333$ & 2.1 & $1.9-3.6$ & 13 & $7.0-16$ \\
\hline II-3 & $\mathrm{F}$ & - & -0.2 & 1,223 & $686-2,019$ & 77 & $106-368$ & 2 & $1.9-3.6$ & 13 & $7.0-16$ \\
\hline II-4 & M & - & -0.1 & 1,406 & $686-2,019$ & 226 & $121-237$ & 2.9 & $1.9-3.6$ & 18 & $7.0-16$ \\
\hline III-4 & $\mathrm{F}$ & 10.5 & -2.9 & 986 & $431-1,892$ & 620 & $132-376$ & 4.7 & $2.0-4.8$ & 24 & $5.6-16$ \\
\hline III-5 & $\mathrm{F}$ & $<10$ & $\mathrm{nl}$ & 854 & $267-1,638$ & 121 & $112-276$ & 2.8 & $2.1-4.2$ & 13 & $2.3-11$ \\
\hline III-6 & $\mathrm{F}$ & 21 & -5 & 854 & $686-2,019$ & 187 & $155-432$ & 2.8 & $2.0-4.2$ & 14 & $7.0-16$ \\
\hline III-8 & M & 16.7 & -5.2 & 534 & $431-1,892$ & 266 & $236-524$ & 3.6 & $2.5-4.8$ & 16 & $5.6-16$ \\
\hline III-9 & $\mathrm{F}$ & 14.8 & -3.25 & 639 & $431-1,892$ & 340 & $217-589$ & 4.5 & $2.2-5.9$ & 23 & $5.6-16$ \\
\hline
\end{tabular}

Patient ID: refer to figure $1 . \mathrm{nl}=$ Normal.

TCTGACTTCATTCTCCGCAGT (exon 11), to generate a PCR product of 709 nucleotides. PCR products were visualized on $2 \%$ agarose gels [15].

\section{Results}

\section{Normal Endocrine Biochemistries Associated with Severe Short Stature}

The GH-IGF-1 axis was initially assessed, since the shared phenotype amongst the affected family members was severe short stature. Serum GH binding protein (GHBP), IGF-1, IGFBP-3 and acid labile subunit (ALS) concentrations were within the reference range for most samples (table 2) and, together with poor growth responses to recombinant $\mathrm{GH}$ therapy, suggested IGF-1 resistance. $\mathrm{Pa}$ tient III- 3 was treated with GH for 6 months at the age of 11 and grew $1.5 \mathrm{~cm}$, while III- 8 was treated with GH for 1 year around the age of 17 and grew $2.9 \mathrm{~cm}$. Patient III- 9 also received GH at the age of 12 and grew approximately $4.4 \mathrm{~cm}$ during the first year of treatment. Targeted gene sequencing of the IGF-1 receptor $(I G F 1 R)$, a known causal gene for IGF-1 resistance, and the IGF1 gene, was unrevealing.

\section{Identification of Novel Intronic ERCC6 Variant by \\ Exome Sequence Analysis}

Whole-exome sequencing analysis was undertaken given the consanguineous pedigree and number of affected individuals in the extended family (fig. 1). In the exome sequencing analysis of the 4 affected relatives and 1 unaffected relative, 17,186 family-specific variants were identi- fied. We filtered out variants present in another unrelated family with a different phenotype who underwent exome sequencing at the same time, as well as variants present in more than $1 \%$ of the population (1,000 genomes and NHLBI Exome Variant Server). We also filtered for a recessive pattern of inheritance given the consanguinity in the proband's family and looked for variants present in both parental chromosomes (homozygous) in the affected individuals but not in the nonaffected family member, III-5. These criteria reduced the number of candidate variants to 2: a presumed splice-site ERCC6 variant and a missense variant in C10orf71, a gene which encodes an uncharacterized protein, also located at chromosome 10q11.23. Although the C10orf71 missense variant and the ERCC6 variant, $c .1992+3 A>G$, demonstrated similar segregation patterns, the novel ERCC6 variant was of particular interest since ERCC6 mutations are causal of Cockayne syndrome, and a defect in ERCC6 could explain some of the clinical features present in the affected family members. We confirmed, by Sanger sequencing, ERCC6 c.1992+3A>G to be homozygous in all affected family members with III- 5 and parents shown to be nonsyndromic carriers (fig. 1).

Given the additional Turner-like phenotypic features present in III-1, III-3 and III-4, we performed a separate analysis of available exome data to assess for other possible recessive genes. We filtered for rare (less than 1\%) or novel homozygous variants that would be present in III- 1 and III-3 but heterozygous or absent in the other family members, and eliminated variants present in another unrelated family. The only potential candidate gene, C15orf59, encodes for an uncharacterized protein, 
Fig. 2. ERCC6 c. $1992+3 A>G$ variant is a splice site defect. DS $=$ Donor site. a Predicted donor site scores are shown associated with exon 9 at the exon-intron junction and the 'putative' site within the exon. The predicted donor site score for ERCC6 c. $1992+3 A>G$ is shown in red. b ERCC6 cDNA from primary fibroblasts, normal (C1) affected family members (III-9, III-8, III-1) and a carrier family member (III-5). Normal RT-PCR-amplified ERCC6 cDNA product is absent in affected individuals homozygous for ERCC6 c.1992 + 3A>G. Smaller, RT-PCR-amplified ERCC6 cDNA products are apparent in the affected and carrier cases. c Sanger sequencing of RTPCR-amplified cDNA products confirmed the use of the alternate splice donor site within exon 9 (underlined; dinucleotide, GT, of putative donor site is boxed) in affected family members. The new mRNA is missing a portion of exon 9 and no longer in frame with the wild-type ERCC6 protein.

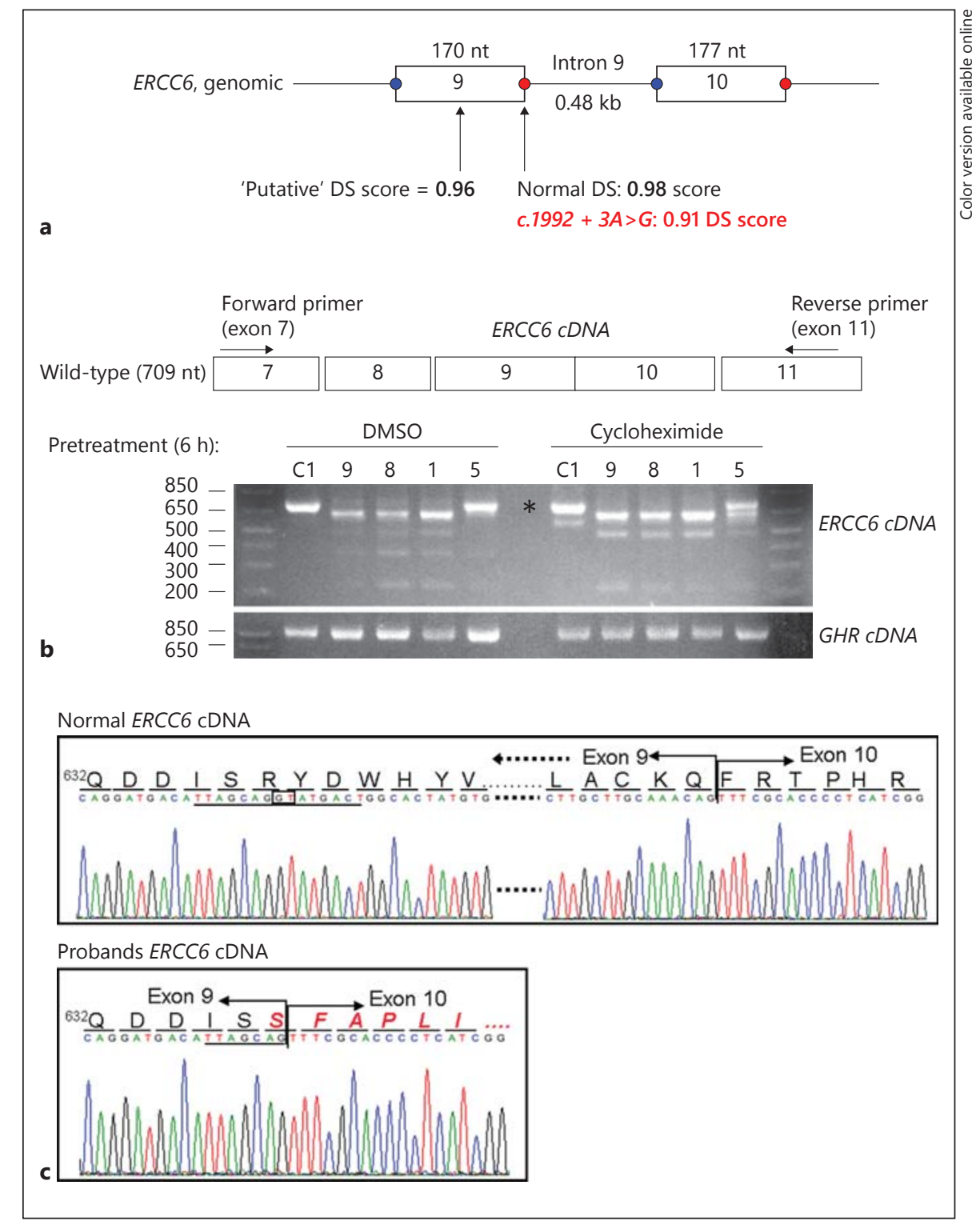

with the variant located in the $3^{\prime}$-untranslated region). The contribution(s) of this variant to the Turner-like phenotype remains unclear.

\section{ERCC6 c. $1992+3 A>G$ Is a Splice Site Variant}

The ERCC6 c.1992 + 3A variant is well conserved amongst species, including rhesus monkeys, dogs, mice, elephants, chickens, and Xenopus (UCSC Genome Browser). Its location immediately downstream of the invariant dinucleotide GT donor splice site of intron 9 suggested it could potentially affect splicing events. Computational analysis employing a splice site prediction tool (http://www.fruitfly.org/seq_tools/splice.html) in- dicated a strong donor site score of 0.98 (out of a maximum of 1.0) for the normal donor site at intron 9, and was 0.91 with the $c .1992+3 A>G$ substitution (fig. 2a). Intriguingly, a putative donor site was recognized within exon 9 that scored a comparably high 0.96 . This raised the question of whether the mild perturbation at the normal donor site might be sufficient to induce splicing events that include the utilization of the putative donor site in exon 9.

To evaluate whether aberrant splicing events occurred, we extracted total RNA from primary fibroblasts derived from 3 of the affected patients and 1 nonsyndromic carrier and, by RT-PCR, compared their ERCC6 cDNA to 
those from normal control fibroblasts. To ensure that as many ERCC6 RNA species as possible were collected, the cells were pretreated with cycloheximide, a potent protease inhibitor that can block mRNA degradation. In figure $2 \mathrm{~b}$, the PCR-amplified portion of the ERCC6 cDNA from exon 7 through exon 11 is shown. From vehicle-treated (DMSO) control fibroblasts, the only PCR product observed was the expected wild-type fragment of around 700 nucleotides in length (fig. 2 b). In contrast, there were multiple PCR products reproducibly detected in fibroblasts from the affected individuals (III-9, III-8, III-1), with a smaller dominant product of about 630 nucleotides. This nucleotide product was even more prominent in cycloheximide-treated patient cells, but was still undetectable in normal cells. Fibroblasts from the unaffected carrier (III-5), as expected, carried both the wild-type as well as the smaller PCR products. Interestingly, PCR amplification of an unrelated cDNA, the GHR cDNA, resulted in the same product for both the affected and normal fibroblasts (fig. 2b), suggesting that the abnormal ERCC6 cDNA profile in affected fibroblasts was not a global phenomenon.

Sequencing of the PCR products confirmed the wildtype ERCC6 cDNA in control fibroblasts and that the dominant approximately 630-nucleotide product in the affected family members corresponded to a splicing event involving the putative donor site in exon 9 (fig. 2c). The aberrant splicing would result in a frameshift with early protein termination (p.Arg637Serfs*34). Altogether, our results indicate that the ERCC6 variant identified in the Turkish family affected the normal splicing pattern, as predicted in silico, and demonstrate how minor changes in the relative strength of the donor sites can have a significant biological impact.

\section{Discussion}

Recent significant advances in genomic technology have the potential to complement clinical diagnosis. Next-generation sequencing can be particularly helpful in cases that elude clinical diagnosis because they are at the milder end of a phenotypic spectrum or confounded by other syndromic features, as was the situation in the present study. Our utilization of next-generation exome sequencing led to the identification of a novel variant in ERCC6, a causal gene for Cockayne syndrome, and resulted in the critical reevaluation of clinical features in our patients. Since the phenotypic range of Cockayne syndrome is broad, it was difficult to make a correct di- agnosis based solely on the clinical features presented by our patients. Recent reports have reviewed the use of clinical exome sequencing in helping with challenging diagnoses across a range of disorders. Based on these reviews, it seems that approximately $25 \%$ of undiagnosed cases suspected of having a genetic disorder can successfully be diagnosed using clinical exome sequencing [17].

We identified 6 individuals in an extended Turkish family who were homozygous for the same ERCC6 c. $1992+3 A>G$ variant. Clinically, the only unifying feature in their presentations was significant short stature, with the 1 affected male (III-8) displaying none of the other syndromic features. The remaining affected patients had variable degrees of photosensitivity and neurological and IQ deficits associated with Cockayne syndrome. As seen in figure 2, the splicing patterns were similar in all of the family members regardless of phenotype. In addition, 3 sisters in one branch of the family (III-1, III-3, III-4) had some Turner-like features, including webbed neck and low posterior hairline, and the postpubertal sisters also presented with symptoms associated with polycystic ovarian syndrome, including hirsutism and hyperandrogenism. The genetic basis for these additional features was independently assessed, but no cause was ascertained. Thus, while the novel ERCC6 mutation was likely to be causal of the Cockayne syndromic features, we suspect that the variable presentations may well be explained by variable splicing efficiencies. The marked phenotypic heterogeneity unrelated to Cockayne syndrome observed in this family, furthermore, may be ascribed to complications of consanguinity and the potential for expression of other recessive traits not captured in our exome analysis.

Consistent with prior reports of patients with Cockayne syndrome [5], the biochemistries of our affected family members, including serum GHBP, IGF-1, IGFBP-3, and ALS (table 2), were notably unremarkable. These relatively normal-to-high levels in the absence of appropriate growth and the poor response to GH therapy are consistent with IGF-1 resistance. The mechanism of this resistance, however, remains unclear.

The homozygous ERCC6 c.1992 + 3A>G splicing variant characterized in our patients is the first described at the ERCC6 exon 9-intron 9 junction. At least 12 ERCC6 splicing mutations have been reported to date [4], of which the majority altered the essential dinucleotides at the donor splice site (GT) or the acceptor splice site (AG), resulting in the inactivation of normal splicing events. Our identified homozygous c. $1992+3 A>G$, in contrast, was not predicted to be inactivating. By analyzing ERCC6 
mRNA species from primary fibroblasts derived from the affected patients, however, abnormal splicing events were observed, in which existing exon 9 sequences were utilized as a preferential donor splice site. The new splicing product lacked a segment of exon 9 and, more importantly, was out of frame, resulting in early protein termination (p.Arg637Serfs*34). Hence, the c.1992 + 3A>G variant, although predicted to only slightly perturb splicing, has a profound impact on normal splicing and, moreover, highlights the existence of a functional, competitive, donor splice site within exon 9. Interestingly, a c.1931A $>\mathrm{G}$ variant was recently identified within this same exonic donor splice site in a diagnosed CS III patient $[4,10]$. The authors claimed (data not shown) that a novel donor site was activated, resulting in the same predicted p.Arg637Serfs*34 truncation. Of note, only 2 other mutations in exon 9 (encoding residues 608-664) have been reported, p.R652* and p.T659Cfs*23 (both predicted truncations), and are associated with CS II subjects who died at the age of 2.5 years [4] and 4.5 years [18], respectively.

It remains unclear whether a truncated CSB protein would be stably expressed from the ERCC6 c.1992+ $3 A>G$ mRNA. The predicted truncated peptide, ERCC6 p.Arg637Serfs*34, would lack most of the central ATPase domain (consisting of 7 helicase motifs of which exon 9 encodes for part of helicase II) and, therefore, would be unable to carry out the DNA-stimulated ATPase activities important for DNA repair and transcriptional regulation. Only the N-terminal domain, with its distinctive acidic motif (residues 356-394) of unknown function, would be retained, but the biological implications of this truncated protein remains to be elucidated. Since a total loss of CSB protein has been associated with a mild phenotype consisting of photosensitivity without neurological findings or short stature [10], it is possible that the truncated predicted ERCC6 variant might be expressed in our patients. However, an absence of CSB has also been associated with a severe phenotype [4], confounding clear genotype-phenotype correlations. Indeed, phenotypic correlation could not be definitively established between missense mutations, nonsense mutations and severity of disease [19]. Interestingly, alternative splicing of ERCC6 involving exon 5 and a domesticated 'PiggyBac' transposable element $P G B D 3$ (encoding for a transposase) residing in intron 5 of ERCC6 generated a CSB-PGBD3 fusion protein that, in the absence of the full-length CBS protein, was proposed to be causal of Cockayne syndrome [20, $21]$. The CSB-PGBD3 fusion protein, however, was not detected in all severe cases of Cockayne syndrome [4].

In conclusion, we describe a new ERCC6 splicing defect causal of Cockayne syndrome. The application of exome sequence analysis was an integral part of the diagnosis given the complexity of phenotypic presentation in the affected family members. The novel splicing defect identified illustrates how a seemingly minor change in the relative strength of a splice site can have significant biological consequences.

\section{Acknowledgments}

We thank the patients and their families for their participation in the study.

This work was supported by National Institutes of Health grants 1K23HD073351 (to A.D.) and 5T32DK007699-32 (to J.S.), as well as the Pediatric Endocrine Society Clinical Scholar Award (to A.D.).

\section{Disclosure Statement}

The authors have no relevant conflicts of interest.

\section{References}

1 Cockayne EA: Dwarfism with retinal atrophy and deafness. Arch Dis Child 1936;11: $1-8$.

2 Moyer DB, Marquis P, Shertzer ME, Burton BK: Cockayne syndrome with early onset of manifestations. Am J Med Genet 1982;13: 225-230.

-3 Nance MA, Berry SA: Cockayne syndrome: review of 140 cases. Am J Med Genet 1992;42: $68-84$.

4 Laugel V, Dalloz C, Durand M, Sauvanaud F, Kristensen U, Vincent MC, et al: Mutation update for the CSB/ERCC6 and CSA/ERCC8 genes involved in Cockayne syndrome. Hum Mutat 2010;31:113-126.

5 Laugel V: Cockayne syndrome: the expanding clinical and mutational spectrum. Mech Ageing Dev 2013;134:161-170.

6 Hashimoto S, Suga T, Kudo E, Ihn H, Uchino M, Tateishi S: Adult-onset neurological degeneration in a patient with Cockayne syndrome and a null mutation in the CSB gene. J Invest Dermatol 2008;128:15971599.

7 Brooks PJ: Blinded by the UV light: how the focus on transcription-coupled NER has distracted from understanding the mechanisms of Cockayne syndrome neurologic disease. DNA Repair (Amst) 2013;12:656671.

8 Lake RJ, Fan H-Y: Structure, function and regulation of CSB: a multi-talented gymnast. Mech Ageing Dev 2013;134:202-211.

9 Venema J, Mullenders LH, Natarajan AT, van Zeeland AA, Mayne LV: The genetic defect in Cockayne syndrome is associated with a defect in repair of UV-induced DNA damage in transcriptionally active DNA. Proc Natl Acad Sci U S A 1990;87:4707-4711. 
10 Horibata K, Iwamoto Y, Kuraoka I, Jaspers NGJ, Kurimasa A, Oshimura M, et al: Complete absence of Cockayne syndrome group B gene product gives rise to UV-sensitive syndrome but not Cockayne syndrome. Proc Natl Acad Sci U S A 2004;101:15410-15415.

11 Li H, Durbin R: Fast and accurate short read alignment with Burrows-Wheeler transform. Bioinformatics 2009;25:1754-1760.

12 McKenna A, Hanna M, Banks E, Sivachenko A, Cibulskis K, Kernytsky A, et al: The Genome Analysis Toolkit: a MapReduce framework for analyzing next-generation DNA sequencing data. Genome Res 2010;20:1297-1303.

13 DePristo MA, Banks E, Poplin R, Garimella KV, Maguire JR, Hartl C, et al: A framework for variation discovery and genotyping using next-generation DNA sequencing data. Nat Genet 2011;43:491-498.

-14 Dauber A, Stoler J, Hechter E, Safer J, Hirschhorn JN: Whole exome sequencing reveals a novel mutation in CUL7 in a patient with an undiagnosed growth disorder. J Pediatr 2013;162:202-204.e1.
15 Akınci A, Rosenfeld RG, Hwa V: A novel exonic GHR splicing mutation (c.784G $>C$ ) in a patient with classical growth hormone insensitivity syndrome. Horm Res Paediatr 2013; 79:32-38.

16 Fang P, Schwartz ID, Johnson BD, Derr MA, Roberts CT, Hwa V, et al: Familial short stature caused by haploinsufficiency of the insulin-like growth factor I receptor due to nonsense-mediated messenger ribonucleic acid decay. J Clin Endocrinol Metab 2009;94: 1740-1747.

17 Yang Y, Muzny DM, Reid JG, Bainbridge MN, Willis A, Ward PA, et al: Clinical wholeexome sequencing for the diagnosis of mendelian disorders. N Engl J Med 2013;369: 1502-1511.
8 Colella S, Nardo T, Mallery D, Borrone C, Ricci R, Ruffa G, et al: Alterations in the CSB gene in three Italian patients with the severe form of Cockayne syndrome (CS) but without clinical photosensitivity. Hum Mol Genet 1999;8:935-941.

19 Mallery DL, Tanganelli B, Colella S, Steingrimsdottir $\mathrm{H}$, van Gool AJ, Troelstra $\mathrm{C}$, et al: Molecular analysis of mutations in the CSB (ERCC6) gene in patients with Cockayne syndrome. Am J Hum Genet 1998;62:77-85.

20 Newman JC, Bailey AD, Fan H-Y, Pavelitz T, Weiner AM: An abundant evolutionarily conserved CSB-PiggyBac fusion protein expressed in Cockayne syndrome. PLoS Genet 2008;4:e1000031.

21 Bailey AD, Gray LT, Pavelitz T, Newman JC, Horibata K, Tanaka K, et al: The conserved Cockayne syndrome B-PiggyBac fusion protein (CSB-PGBD3) affects DNA repair and induces both interferon-like and innate antiviral responses in CSB-null cells. DNA Repair (Amst) 2012;11:488-501. 